\title{
Image Feature
}

National Cancer Institute

\section{Source}

National Cancer Institute. Image Feature. NCI Thesaurus. Code C70838.

In image processing, a piece of information which is relevant for solving the

computational task related to a certain application, either specific structures in the image itself (e.g., simple structures such as points or edges to more complex structures such as objects) or the result of a general neighborhood operation (feature extractor or feature detector) applied to the image. 